\title{
Gas-phase Electronic Spectra of Coronene and Corannulene Cations
}

\author{
F.-X. Hardy, Corey A. Rice, and John P. Maier \\ Department of Chemistry, University of Basel, Klingelbergstrasse 80, 4056 Basel, Switzerland; j.p.maier@unibas.ch \\ Received 2016 October 20; revised 2017 January 3; accepted 2017 January 4; published 2017 February 7
}

\begin{abstract}
Gas-phase electronic spectra of the coronene $\left(\mathrm{C}_{24} \mathrm{H}_{12}{ }^{+}\right)$and corannulene $\left(\mathrm{C}_{20} \mathrm{H}_{10}{ }^{+}\right)$cations complexed with helium have been recorded in a quadrupole ion trap at $5 \mathrm{~K}$ by photodissociation. The electronic spectrum of $\mathrm{C}_{20} \mathrm{H}_{10}{ }^{+}$with two helium atoms was also measured to estimate the perturbation. This method is sufficient for an astronomical comparison because the shift due to the weakly bound helium is on the order of $0.2 \AA . \mathrm{C}_{24} \mathrm{H}_{12}{ }^{+}$-He has the origin band of the $\mathrm{A}^{2} \mathrm{E}_{1 g} \leftarrow X^{2} \mathrm{E}_{2 u}$ transition at $9438.3 \AA$ and that to a much higher state $\mathrm{D}_{3} \leftarrow X^{2} \mathrm{E}_{2 u}$ at $4570 \AA$. The corannulene cation is subject to a Jahn-Teller distortion in the electronic ground state, leading to the $3^{2} \mathrm{~A}^{\prime} \leftarrow \mathrm{X}^{2} \mathrm{~A}^{\prime \prime}$ and $3^{2} \mathrm{~A}^{\prime \prime} \leftarrow \mathrm{X}^{2} \mathrm{~A}^{\prime}$ transitions with origin band maxima when complexed with helium at 5996.1 and 5882.6 $\AA$. These absorptions lie in a region where there is a congestion of diffuse interstellar bands (DIBs). However, the recorded features have no match with astronomical observations, removing coronene and corannulene cations and probably other aromatic hydrocarbons of this size as possible carriers of the DIBs.
\end{abstract}

Key words: astrochemistry - line: identification - methods: laboratory: molecular - techniques: spectroscopic

\section{Introduction}

Diffuse interstellar bands (DIBs) are attributed to gas-phase molecules partly because of the resemblance of unresolved rotational contours recorded in astronomical spectra at different line of sights. Carbon-containing species, such as polycyclic aromatic hydrocarbons (PAHs), have been proposed as possible candidates (Léger \& d'Hendecourt 1985; Van der Zwet \& Allamandola 1985; Steglich et al. 2011). Carbon has a tendency to form complex molecules and has a large galactic abundance of $0.6 \%$ that of hydrogen. PAHs composed of more than 40-50 carbon atoms are photostable and are less likely to fragment from the UV flux in the interstellar medium (ISM; Tielens 2008). Unidentified infrared emission bands, assigned to vibrational de-excitation of aromatic compounds (Sellgren 1984; Mackie et al. 2015), show that ionic and neutral PAHs could be widespread in the ISM and possibly contain approximately $20 \%$ of the carbon in space.

DIBs are observed toward different binary star systems in the 4000-9000 A region. To identify a specific molecule as a potential carrier, its electronic absorption spectrum is usually obtained in a rare-gas matrix and, subsequently, in the gas phase at low temperatures. In the past, spectra have been recorded for dozens of carbon chains and rings in the gas phase, neutral and cationic. None could be correlated with DIBs, and only upper limits of the column densities were inferred (Zack \& Maier 2014). PAH cations have been previously studied in rare-gas matrices (Mattioda et al. 2005); however, they remain a challenge for gas-phase laboratory observations (Pino et al. 2014; Salama \& Ehrenfreund 2014). Most often gas-phase electronic spectra were recorded by monitoring the photofragmentation of the molecule as a function of the wavelength, because large ionic species ( $>20$ carbon atoms) have low quantum efficiencies for fluorescence and are not in high enough densities for direct absorption. Electronic spectra could not be recorded in the gas phase for large or even medium-size $\mathrm{PAH}^{+} \mathrm{s}$ (Salama \& Ehrenfreund 2014). Protonated PAHs ( $\left.{ }^{+} \mathrm{PAHs}\right)$ were also proposed as candidates when selected ion flow tube experiments showed a high chemical reactivity of $\mathrm{PAH}^{+} \mathrm{s}$ with hydrogen (Snow et al. 1998; Pathak \& Sarre 2008). It was concluded that if $\mathrm{PAH}^{+} \mathrm{s}$ were present in the ISM, protonation was likely to deplete their abundance. Theoretical calculations also showed that many electronic transitions of $\mathrm{H}^{+}$PAHs (20-50 C atoms) fall in the DIB range. However, recent gas-phase spectra on medium-size $\mathrm{H}^{+} \mathrm{PAHs}$ (16-24 carbons) found no match with DIBs (Hardy et al. 2013; Patzer et al. 2013; Rice et al. 2014; Noble et al. 2015). It is now thought that if $\mathrm{H}^{+}$PAHs are in the ISM, they must contain $>40$ carbon atoms.

Coronene $\left(\mathrm{C}_{24} \mathrm{H}_{12}\right)$ and corannulene $\left(\mathrm{C}_{20} \mathrm{H}_{10}\right)$ are two members of the PAH family. $\mathrm{C}_{24} \mathrm{H}_{12}$ is a highly symmetric peri-condensed PAH $\left(\mathrm{D}_{6 h}\right)$, while $\mathrm{C}_{20} \mathrm{H}_{10}\left(\mathrm{C}_{5 v}\right)$ presents some unique properties for astronomical detection because of its dipole moment of $2.1 \mathrm{D}$ (Lovas et al. 2005). Structurally, $\mathrm{C}_{20} \mathrm{H}_{10}$ is the smallest $\mathrm{PAH}$ adopting a bowl shape, given by its central five-membered ring and being a third of $\mathrm{C}_{60}$. Recently, laboratory spectra confirmed that gas-phase $\mathrm{C}_{60}^{+}$is a DIB carrier (Campbell et al. 2015, 2016a), making $\mathrm{C}_{20} \mathrm{H}_{10}$ of particular interest as a possible building block of $\mathrm{C}_{60}$.

A method to obtain the electronic spectrum of larger ions consists of attaching a rare-gas atom and monitoring the photodissociation of the complex (Patzer et al. 2013). A number of electronic spectra were obtained using argon as a messenger, but the perturbations on the absorption bands are too large to make a reliable comparison with astronomical data (Pino et al. 2014). A weakly bound helium atom has significantly less distortion on the observed features in the electronic spectrum (Bieske et al. 1992; Chalyavi et al. 2015; Campbell et al. 2016b). The efficient production of helium complexes requires an ion trap for confinement of the charged species and to increase the interaction time with the buffer gas (Johnson et al. 2014; Roithová et al. 2016).

If present in the ISM, coronene and corannulene would also be ionized by UV radiation. In this article the approach adopted to obtain gas-phase spectra of coronene and corannulene cations under conditions in diffuse interstellar clouds with the internal degrees of freedom equilibrated to approximately $10 \mathrm{~K}$ is described and the laboratory data are compared with the astronomical observations. Measurements involve the use of a 
radio frequency (rf) trap, collisional cooling with $5 \mathrm{~K}$ helium, and a laser excitation-dissociation scheme.

\section{Experimental Method}

The experimental setup has been described (Hardy et al. 2015). Cations are created in electron impact from vapors of coronene or corannulene heated to $\approx 150^{\circ} \mathrm{C}$. Ions from the source are accumulated in a hexapole, where collisions with residual gas from the source narrow the kinetic energy distribution. The exit potential is lowered, and cations are released into a quadrupole mass selector, discriminating $\mathrm{C}_{24} \mathrm{H}_{12}{ }^{+}$or $\mathrm{C}_{20} \mathrm{H}_{10}{ }^{+}$. The ion beam is deflected $90^{\circ}$, injected into an rf-only octupole, and transported to a cryogenic ion trap, confining positive species in the radial and axial directions.

A $36 \mathrm{~mm}$ long wire quadrupole trap (Jašík et al. 2013) is mounted on the second stage of a closed-cycle helium cryostat at $4 \mathrm{~K}$ and filled with $\approx 10^{5}$ ions per cycle. The sequence is $200 \mathrm{~ms}$ for injecting cations into a 4-pole, followed by $800 \mathrm{~ms}$ of cooling, while triggering laser irradiation at $10 \mathrm{~Hz}$. Within the cryogenic environment $\mathrm{C}_{24} \mathrm{H}_{12}{ }^{+}-\mathrm{He}$ or $\mathrm{C}_{20} \mathrm{H}_{10}{ }^{+}-\mathrm{He}$ complexes are produced in a quadrupole. A piezo-valve introduced helium buffer gas into the trap from the beginning of the filling period, achieving after a few milliseconds a helium density of $\approx 5 \times 10^{15} \mathrm{~cm}^{-3}$. This leads to one collision of a $\mathrm{PAH}^{+}$with a helium atom per microsecond. At the end of a cycle, the buffer gas is pumped out before the ions are ejected, for detecting photofragmentation products. Positive species exiting the trap are collimated by electrodes, deflected $90^{\circ}$, and transported into the second 4-pole mass filter. The latter selected $m / z=254$ or 304 , and a depletion in $m / z$ is monitored as a function of laser wavelength $(R=300,000)$ to record an electronic spectrum.

\section{Results and Discussion}

The electronic spectrum of $\mathrm{C}_{24} \mathrm{H}_{12}{ }^{+}$in the 4000-10000 region was first recorded in a $4 \mathrm{~K}$ neon matrix (Ehrenfreund et al. 1992). In this experiment two absorptions at 4590 and $9465 \AA$ A were observed with experimentally estimated oscillator strengths of $\approx 0.01$ and 0.002 (Ehrenfreund et al. 1992). Subsequent gas-phase investigations by multiphoton dissociation (MPD; Useli-Bacchitta et al. 2010) led to the observations of two bands at 4570 and $4320 \AA$ and assigned to higher excitations $\mathrm{D}_{11,10} \leftarrow X{ }^{2} \mathrm{E}_{2 u}$ of $\mathrm{C}_{24} \mathrm{H}_{12}{ }^{+}$. In that experiment, the internal temperature of the ions was $35 \mathrm{~K}$, and the MPD process leads to broadening effects of the bands due to the heating of the ion ensemble.

Two absorptions were observed in the gas phase by recording a depletion of the $\mathrm{C}_{24} \mathrm{H}_{12}{ }^{+}-\mathrm{He}$ complexes (Figure 1, black). The $\mathrm{A}^{2} \mathrm{E}_{1 g} \leftarrow X{ }^{2} \mathrm{E}_{2 u}$ electronic transition of $\mathrm{C}_{24} \mathrm{H}_{12}{ }^{+}-\mathrm{He}$ consists of one band at $9438.3 \pm 0.2 \AA$ blueshifted to the neon matrix value by $\approx 30 \AA$. The observed band has a width of $1.2 \AA$, and no rotational structure is apparent with the resolving power of the laser $(R=94,000)$. This corresponds to an excited lifetime of a few picoseconds. This is in agreement with the threshold photoelectron spectrum (TPES), having a band maximum at $9500 \pm 200 \AA$ (Bréchignac et al. 2014). If the excited state is split into two separate J-T levels, the upper states would share the intensity and have the same band strength in the electronic spectrum. This is not observed in the presented measurements. In the first excited state, the inferred J-T effect would then be less than the laser bandwidth. In the TPES of coronene, only a vibrational progression is seen, but no splitting of $\approx 100 \mathrm{~cm}^{-1}$.

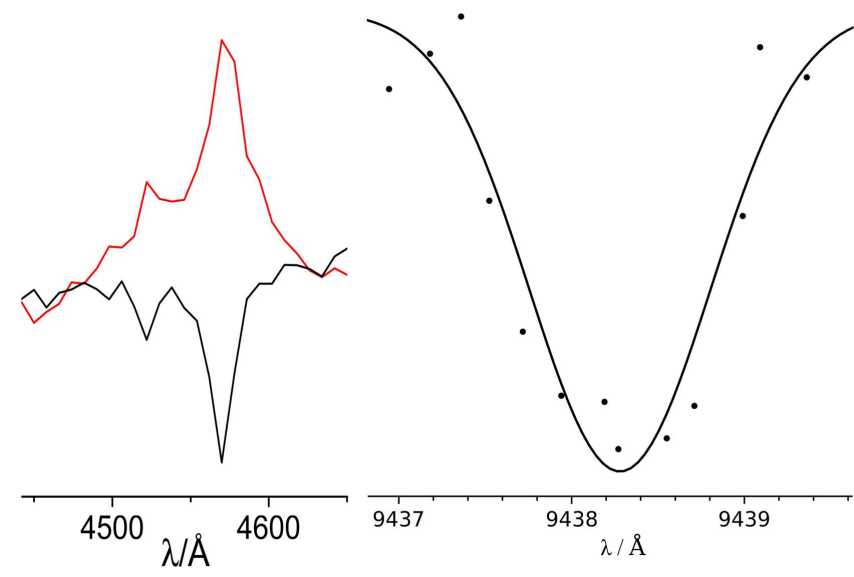

Figure 1. Gas-phase electronic spectra of the coronene cation, $\mathrm{C}_{24} \mathrm{H}_{12}{ }^{+}$, complexed with helium recorded with a laser of $1 \AA$ bandwidth in the visible (left) and $0.1 \AA$ in the near-infrared (right). The absorptions measured the depletion of the $\mathrm{C}_{24} \mathrm{H}_{12}{ }^{+}-\mathrm{He}$ complexes (black), and that in red was observed by monitoring the mass channel of $\mathrm{C}_{24} \mathrm{H}_{12}{ }^{+}$. The left panel is the $\mathrm{D}_{3} \leftarrow X^{2} \mathrm{E}_{2 u}$ electronic transition of $\mathrm{C}_{24} \mathrm{H}_{12}{ }^{+}-\mathrm{He}$, and the right panel is that of the origin band of $\mathrm{A}^{2} \mathrm{E}_{1 g} \leftarrow X{ }^{2} \mathrm{E}_{2 u}$.

The $\mathrm{D}_{3} \leftarrow X^{2} \mathrm{E}_{2 u}$ electronic transition at $4570 \pm 10 \AA$ shows no vibrational structure and is blueshifted compared to the neon matrix absorption by $\approx 20 \AA$. In TPES the band maximum is shifted similarly as in crystalline neon and located at $4542 \AA$. The red trace on the left of Figure 1 was recorded by monitoring the depletion of the complexes $(m / z=304)$, and that in black was measured using $m / z=300$. This confirms that ions from $m / z=304$ boil off the helium atom and do not fragment further into other dissociation channels. The absorption band maximum wavelength is in agreement with the MPD data, and any spectral broadening is due to the excited-state lifetime $(\approx 50 \mathrm{fs})$. The widths of the bands in the MPD experiment are broader owing to the higher internal temperature of the ion.

The electronic spectrum of $\mathrm{C}_{20} \mathrm{H}_{10}+$ in the $6000 \AA$ region was first recorded in a $6 \mathrm{~K}$ neon matrix after mass-selected deposition (Rice et al. 2015). The gas-phase electronic spectrum of $\mathrm{C}_{20} \mathrm{H}_{10}{ }^{+}-\mathrm{He}$ at temperatures $<10 \mathrm{~K}$ is presented in Figure 2. $\mathrm{C}_{20} \mathrm{H}_{10}{ }^{+}$has two Jahn-Teller structures in the ground state, leading to two origin bands at 5996.0 and 5882.6 $\AA$. The spectrum is similar to the one recorded in a neon matrix from which it is blueshifted by $16 \AA$. The $3^{2} \mathrm{~A}^{\prime} \leftarrow X^{2} \mathrm{~A}^{\prime \prime}$ and $3^{2} \mathrm{~A}^{\prime \prime} \leftarrow X^{2} \mathrm{~A}^{\prime}$ transitions were recorded first with a lowerresolution laser with a $1 \AA$ bandwidth (Figure 2). Absorption features $\mathrm{A}$ and $\mathrm{D}$ in Figure 2 (insets) were also measured with $0.02 \AA$ resolution. An unresolved rotational contour can be observed, which resembles a perpendicular transition of an asymmetric-top molecule in A. This is due to the strong $Q$ branch in the rotational profile; however, this is not the case for $\mathrm{D}$, where the $Q$-branch is weaker and blended into the unresolved rotational profile.

To estimate the perturbation of tagging, the spectrum with two helium atoms attached was recorded with a resolution of $0.02 \AA$ (Figure 2). For the origin band at $5882.6 \AA$, the FWHM increases from 0.5 to $0.7 \AA$ with no significant shift. In the case of the origin band at $5996.0 \AA$, the FWHM increases from 0.5 to $0.6 \AA$ with a redshift of $0.1 \AA$. The excited-state lifetime can be estimated to be a few picoseconds. The absorption band maximum of $\mathrm{C}_{20} \mathrm{H}_{10}{ }^{+}$itself can be expected to be blueshifted by $\approx 0.1 \AA$ with respect to the given wavelength and possibly 


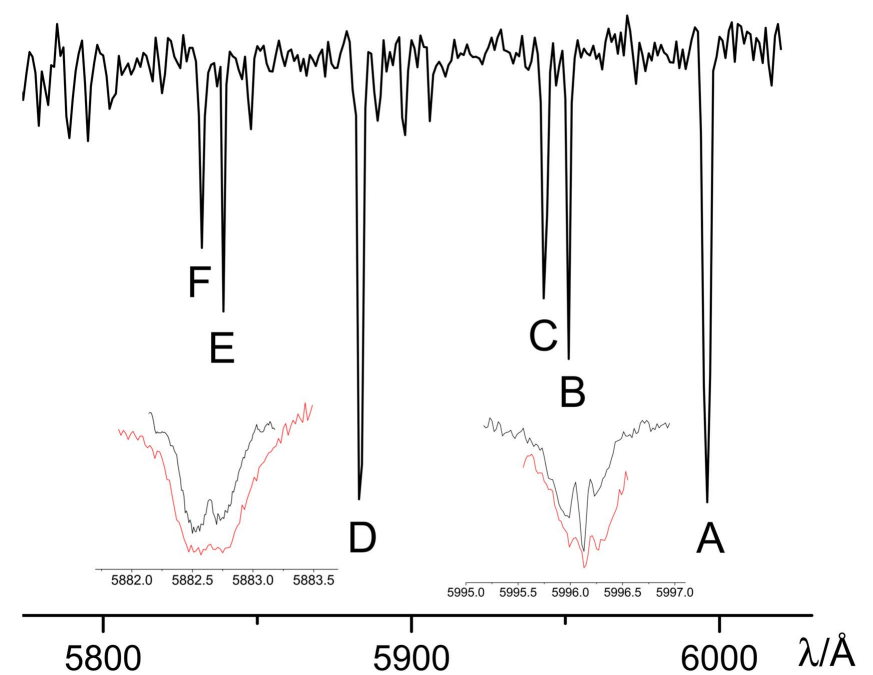

Figure 2. Gas-phase electronic spectrum of the corannulene cation, $\mathrm{C}_{20} \mathrm{H}_{10}{ }^{+}$, complexed with helium recorded with a laser of $1 \AA$ bandwidth $(200 \mu \mathrm{J} /$ pulse $)$. Two Jahn-Teller structures in the electronic ground state of $\mathrm{C}_{20} \mathrm{H}_{10}{ }^{+}$lead to the $3^{2} \mathrm{~A}^{\prime} \leftarrow \mathrm{X}^{2} \mathrm{~A}^{\prime \prime}(\mathrm{A}, \mathrm{B}, \mathrm{C})$ and $3^{2} \mathrm{~A}^{\prime \prime} \leftarrow \mathrm{X}^{2} \mathrm{~A}^{\prime}$ (D, E, F) transitions. Bands $\mathrm{A}$ and $\mathrm{D}$ were measured with a laser power of $50 \mu \mathrm{J} /$ pulse and a resolution of $0.02 \AA$ (black and red insets, respectively). These two absorptions were recorded with one (black) and two (red) helium atoms attached to the corannulene cation.

have a narrower FWHM. Previously, the electronic spectra of $\mathrm{C}_{60}{ }^{+}-\mathrm{He}_{1,2,3}$ were measured to determine the shift induced by tagging and concluded to be $0.7 \AA$ per helium atom (Campbell et al. 2016b). This is slightly larger than for $\mathrm{C}_{20} \mathrm{H}_{10}{ }^{+}-\mathrm{He}_{1,2}$. However, these shifts are smaller than the typical uncertainty in the astronomical data, and thus such results can be used for identifying DIB candidates.

A comparison was made for bands A and D (Figure 2) with DIB measurements toward HD 183143 (Hobbs et al. 2009) and HD 204827 (Hobbs et al. 2008). Though absorption A is similar in position and width to a DIB around 5995.9 $\AA$, that of $\mathrm{D}$ is not observed. In addition, the less intense bands in the electronic spectrum of $\mathrm{C}_{20} \mathrm{H}_{10}{ }^{+}$could not be matched with any DIB. Given a helium perturbation of up to $0.7 \AA$, the absorptions of $\mathrm{C}_{24} \mathrm{H}_{12}{ }^{+}-\mathrm{He}$ at 9438.3 and $4570 \AA$ were also not present in the cataloged DIB data from HD 183143 and HD 204827. The conclusion is that corannulene and coronene cations are not DIB carriers, and that their column density is below the detection limit of $10^{12} \mathrm{~cm}^{-2}$.

\section{Conclusions}

The objective of this paper was to determine experimental wavelengths and bandwidths of the transitions to the lowest excited electronic state of coronene and corannulene cations at an internal temperature of $\approx 10 \mathrm{~K}$, similar to the ISM. The presented gas-phase electronic transitions of $\mathrm{C}_{24} \mathrm{H}_{12}{ }^{+}$and $\mathrm{C}_{20} \mathrm{H}_{10}{ }^{+}$provide a means of spectroscopic identification in the ISM or planetary atmospheres. These species have absorptions that lie in the DIB range ( 4000-9000 $\AA$ ) and can be compared to astronomical data. The results were all negative, having an estimated upper limit of the column density of $<10^{12} \mathrm{~cm}^{-2}$ for $\mathrm{C}_{24} \mathrm{H}_{12}{ }^{+}$and $\mathrm{C}_{20} \mathrm{H}_{10}{ }^{+}$.

If a $\mathrm{PAH}^{+}$has a near-infrared transition, there are excitations to higher states in the visible region as in the case of $\mathrm{C}_{24} \mathrm{H}_{12}{ }^{+}$. These should be considered only if the FWHM of the absorption is comparable to the DIBs $(0.1-10 \AA)$, which corresponds to excited-state lifetimes of 50 to $0.2 \mathrm{ps}$. For $\mathrm{C}_{24} \mathrm{H}_{12}{ }^{+}$the observed visible band near $4570 \AA$ has an FWHM larger than this constriction. On the other hand, the $3^{2} \mathrm{~A}^{\prime} \leftarrow X^{2} \mathrm{~A}^{\prime \prime}$ and $3^{2} \mathrm{~A}^{\prime \prime} \leftarrow X^{2} \mathrm{~A}^{\prime}$ absorptions of $\mathrm{C}_{20} \mathrm{H}_{10}{ }^{+}$have narrower bandwidths. Models predict $\mathrm{PAH}^{+} \mathrm{s}$ of this size to be dehydrogenated in diffuse clouds (Le Page et al. 2003). The approach invoked here could be utilized to obtain the electronic spectra of larger or dehydrogenated $\mathrm{PAH}^{+} \mathrm{s}$.

This work was supported by the European Research Council (ERC-AdG-ElecSpecIons:246998). The Siegel Group at the University of Zurich, especially A. Butterfield, is thanked for the corannulene sample preparation.

\section{References}

Bieske, E. J., Soliva, A. M., Friedmann, A., \& Maier, J. P. 1992, JChPh, 96, 28 Bréchignac, P., Garcia, G. A., Falvo, C., et al. 2014, JChPh, 141, 164325 Campbell, E. K., Gerlich, D., Holz, M., \& Maier, J. P. 2015, Natur, 523, 322 Campbell, E. K., Holz, M., Maier, J. P., et al. 2016a, ApJ, 822, 17

Campbell, E. K., Holz, M., \& Maier, J. P. 2016b, ApJL, 826, L4 Chalyavi, N., Catani, K., Sanelli, J., et al. 2015, MolPh, 113, 2086 Ehrenfreund, P., d'Hendecourt, L., Verstraete, L., et al. 1992, A\&A, 259, 257 Hardy, F.-X., Gause, O., Rice, C. A., \& Maier, J. P. 2013, ApJL, 778, L30 Hardy, F.-X., Rice, C., Gause, O., \& Maier, J. 2015, JPCA, 119, 1568 Hobbs, L., York, D., Thorburn, J., et al. 2009, ApJ, 705, 32 Hobbs, L. M., York, D. G., Snow, T. P., et al. 2008, ApJ, 680, 1256 Jašík, J., Žabka, J., Roithová, J., \& Gerlich, D. 2013, IJMSp, 354, 204 Johnson, C. J., Wolk, A. B., Fournier, J. A., et al. 2014, JChPh, 140, 221101 Le Page, V., Snow, T. P., \& Bierbaum, V. M. 2003, ApJ, 584, 316 Léger, A., \& d'Hendecourt, L. 1985, A\&A, 146, 81

Lovas, F. J., McMahon, R. J., Grabow, J.-U., et al. 2005, JAChS, 127, 4345

Mackie, C. J., Peeters, E., Jr., \& Cami, J. 2015, ApJ, 799, 131

Mattioda, A., Hudgins, D., \& Allamandola, L. 2005, ApJ, 629, 1188

Noble, J., Dedonder, C., \& Jouvet, C. 2015, A\&A, 577, A79

Pathak, A., \& Sarre, P. J. 2008, MNRAS, 391, L10

Patzer, A., Schutz, M., Jouvet, C., \& Dopfer, O. 2013, JPCA, 117, 9785

Pino, T., Féraud, G., Bréchignac, et al. 2014, in Proc. IAU Symp. 297, The Diffuse Insterstellar Bands (Cambridge: Cambridge Univ. Press), 247

Rice, C., Hardy, F.-X., Gause, O., \& Maier, J. 2014, JPCL, 5, 942

Rice, C. A., Hardy, F.-X., Gause, O., \& Maier, J. P. 2015, ApJL, 812, L4

Roithová, J., Gray, A., Andris, E., et al. 2016, AcChR, 49, 223

Salama, F., \& Ehrenfreund, P. 2014, in Proc. IAU Symp. 297, The Diffuse Interstellar Bands (Cambridge: Cambridge Univ. Press), 364

Sellgren, K. 1984, ApJ, 277, 623

Snow, T. P., Le Page, V., Keheyan, Y., \& Bierbaum, V. M. 1998, Natur, 391,259

Steglich, M., Bouwman, J., Huisken, F., \& Henning, T. 2011, ApJ, 742, 2

Tielens, A. G. G. M. 2008, ARA\&A, 46, 289

Useli-Bacchitta, F., Bonnamy, A., Mulas, G., et al. 2010, CP, 371, 16

Van der Zwet, G., \& Allamandola, L. 1985, A\&A, 146, 76

Zack, L. N., \& Maier, J. P. 2014, Chem. Soc. Rev., 43, 4602 